\title{
Uso Antenatal de Corticosteróide e Condiçōes de Nascimento de Pré-termos nos Hospitais da Rede Brasileira de Pesquisas Neonatais
}

\author{
Antenatal Corticosteroid Use and Birth Conditions of Preterm Infants at Hospitals Belonging to \\ the Brazilian Network of Neonatal Research
}

Rede Brasileira de Pesquisas Neonatais*

\section{RESUMO}

\begin{abstract}
Objetivos: avaliar o uso de corticosteróide antenatal (CA) nas mães e as suas repercussões nas condições de nascimento das crianças nascidas pré-termo nas oito unidades neonatais universitárias da Rede Brasileira de Pesquisas Neonatais.

Métodos: estudo observacional do tipo coorte prospectivo. Foram avaliadas 463 gestantes com idade gestacional (IG) entre 23 e 34 semanas e seus 514 recém-nascidos no periodo de 1 de agosto a 31 de dezembro de 2001. Os dados foram obtidos por entrevista materna, análise de prontuário e acompanhamento dos recém-nascidos, e analisados pelos testes do $\chi^{2}$, MannWhitney, ANOVA e regressão logística múltipla, com nivel de significância de 0,05.

Resultados: 60,1\% (282/463) das gestantes (variação de 12,5 a 87,3\% entre as unidades) receberam ao menos uma dose de CA. A freqüência do uso de CA foi diretamente associada ao número de consultas de pré-natal, hipertensão materna e uso antenatal de tocoliticos. Quando as gestantes foram tratadas, os RN apresentaram maior peso de nascimento (1379 \pm 421 vs $1244 \pm 543 \mathrm{~g}$ ), idade gestacional mais avançada (30,9 $\pm 2,0$ vs 29,5 $\pm 3,5$ semanas), melhores valores do escore de Apgar no $1^{\circ}$ e $5^{\circ}$ minuto e menor freqüência de intervenção na sala de parto. O uso de CA, a IG e o fato de ser pequeno para a IG melhoraram, de forma independente, as condições de nascimento.

Conclusões: o uso de CA, na maioria dos centros, foi feito em freqüência abaixo da desejada e em metade das vezes de forma inadequada. O tratamento foi mais aplicado a mães com melhor acompanhamento durante o pré-natal e foi associado com melhores condições de nascimento.
\end{abstract}

PALAVRAS-CHAVE: Corticosteróide antenatal. Prematuridade. Baixo peso. Pré-termo.

Correspondência:

Francisco Eulógio Martinez

Departamento de Puericultura e Pediatria

Faculdade de Medicina de Ribeirão Preto - USP

Av Bandeirantes, 3900.

14049-900 - Ribeirão Preto - SP

Fone: (16) 602-2808

Fax: (16) 602-2700

e-mail: femartin@fmrp.usp.br

* Francisco Eulógio Martinez, Marisa Márcia MussiPinhata, Nelson José Linhares, Sérgio Marba, Abimael A Neto, Renato Procianoy, Natacha T. Uchoa, José Maria Andrade Lopes, Olga Bomfim, Cléa R Leone, Lílian S R Sadeck, Flávio Adolfo Costa Vaz, Ruth Guinsburg, Maria Fernanda B Almeida, Joice Fabíola Meneguel, Milton Miyoshi, Renato M Fiori, Tatiana T Garcia, Jorge H Luz, Cleide Enoir Petean Trindade, Maria Regina Betlin

\section{Introdução}

Há cerca de 30 anos, Liggins e Howie ${ }^{1}$ demonstraram que a administração de corticosteróide a ovelhas prenhes acarretava o amadurecimento pulmonar dos cordeiros nascidos prematuramente. A seguir desenharam estudo randomizado em humanos, no qual avaliaram o efeito da administração materna de betametasona. Com duas doses de 12 $\mathrm{mg}$ por via intramuscular com intervalo de $24 \mathrm{~h}$ entre elas, os autores foram capazes de demonstrar diminuição na incidência de sindrome do desconforto respiratório e na mortalidade entre pré-termos nascidos com menos de 34 semanas de gestação ${ }^{1}$. 
Esses achados estimularam a realização de vários estudos, nas últimas três décadas, sobre o uso antenatal de corticosteróide nas gestações em risco de término prematuro. Apenas na base de dados Medline podem ser detectados mais de 2200 artigos em língua inglesa sobre o uso de corticosteróide para a maturação pulmonar fetal. Nos dias atuais estão disponiveis vários resultados de consenso e estudos de meta-análise de ensaios randomizados que demonstram que essa conduta é eficaz e deve estar incorporada ao arsenal terapêutico2-4. O uso antenatal de corticosteróide mostrou-se capaz de diminuir a ocorrência de síndrome da dificuldade respiratória, de hemorragia peri e intraventricular e da mortalidade neonatal entre os pré-termos ${ }^{5}$.

A despeito dos benefícios já demonstrados, o uso do corticosteróide antenatal não parece ser conduta terapêutica adequadamente disseminada no nosso país. Como exemplo pode-se citar o relato de sua administração em cerca de metade das gestantes que vieram a ter seu filho prematuramente em uma maternidade-escola ${ }^{6}$, ou pior, a irrisória taxa de $4 \%$ para os nascidos com menos de 34 semanas em sete maternidades públicas não universitárias, de níveis secundário e terciário da cidade do Rio de Janeiro ${ }^{7}$.

No presente estudo avaliamos, de forma prospectiva, o uso de corticosteróide antenatal nas mães de crianças nascidas com idade gestacional inferior a 34 semanas nas oito unidades neonatais universitárias pertencentes à Rede Brasileira de Pesquisas Neonatais e analisamos a repercussão do tratamento nas condições de nascimento dos pré-termos.

\section{Métodos}

Participaram do estudo observacional tipo coorte prospectivo todos os neonatos com idade gestacional superior a 23 semanas e inferior a 35 semanas nascidos nas maternidades das oito unidades neonatais da Rede Brasileira de Pesquisas Neonatais no período de 1 de agosto a 31 de dezembro de 2001, e suas mães. Esta rede é composta pelas seguintes instituições, em ordem alfabética: Fundação Oswaldo Cruz (FIOCRUZ)/Instituto Fernandes Figueira; Pontificia Universidade do Rio Grande do Sul (PUCRS)/Hospital São Lucas; Universidade Estadual Paulista (UNESP)/ Faculdade de Medicina de Botucatu Universidade Estadual de Campinas (UNICAMP)/Centro de Atenção Integral à Saúde da Mulher (CAISM); Universidade Federal do Rio Grande do Sul (UFRGS) /Hos- pital de Clínicas de Porto Alegre; Universidade Federal de São Paulo (UNIFESP)/Escola Paulista de Medicina (EPM) / Hospital São Paulo; Universidade de São Paulo (USP/SP)/ Hospital das Clínicas de São Paulo/Berçário Anexo à Maternidade e Universidade de São Paulo (USP/RP)/Hospital das Clínicas de Ribeirão Preto.

Individualmente, cada instituição teve o protocolo de estudo aprovado pelo seu respectivo Comitê de Ética em Pesquisa como estudo multicêntrico.

Foram excluídos os recém-nascidos com anomalias congênitas maiores ${ }^{8}$ ou sindromes cromossômicas. Também não foram considerados para estudo os neonatos cujas mães receberam corticosteróide antenatal para outra finalidade que não a indução da maturação fetal, por exemplo, para o tratamento de doenças crônicas como asma brônquica e colagenoses, entre outras.

Para cada nascimento de criança elegivel a participar da pesquisa foi preenchida uma ficha de protocolo, enquanto a mãe estava internada. As variáveis maternas e neonatais foram obtidas por meio da revisão dos prontuários médicos, do cartão de pré-natal e, quando possivel, da pasta de pré-natal, além de entrevista com a mãe. Os dados foram coletados após a assinatura do Termo de Consentimento Livre e Esclarecido pela mãe. Foram consideradas as seguintes características maternas: idade, gestações anteriores, filhos vivos, número de consultas de pré-natal, doenças durante a atual gestação e uso de tocolíticos e de corticosteróide antenatal, sendo registrados o número de doses, o tipo de droga e a data e horário das administrações.

Considerou-se que o uso de corticosteróide foi adequado quando foram aplicadas por via intramuscular duas doses de betametasona (12 $\mathrm{mg} /$ dose) ou quatro doses de dexametasona (6 mg/ dose). O tempo decorrido entre a última aplicação da droga e o nascimento deveria ser no mínimo de $12 \mathrm{~h}$ e no máximo de 7 dias $^{2}$.

Com relação aos recém-nascidos, foram registrados o tipo e horário do parto, idade gestacional, adequação para a idade gestacional, Apgar de $1^{\circ}$ e $5^{\circ}$ minuto e necessidade de procedimentos de reanimação na sala de parto.

A idade gestacional foi registrada em semanas, determinada por estimativa obstétrica, de acordo com a data da última menstruação, dados ultra-sonográficos ou exame neonatal pelo método New Ballard ${ }^{9}$, que é capaz de detectar melhor as crianças com menor idade gestacional. A idade gestacional definitiva foi considerada aquela calculada pela data da última menstruação ou pela ultra-sonografia fetal precoce quando a diferença 
entre esta data e a determinada pelo exame do recém-nascido era inferior a uma semana. Se a diferença fosse maior que uma semana, era considerada a idade gestacional calculada pelo método de New Ballard.

A adequação do crescimento intra-uterino foi classificada por meio da curva de crescimento intra-uterino de Alexander et al. ${ }^{10}$. Considerou-se como sendo adequado para a idade gestacional o recém-nascido com peso de nascimento entre os percentis 10 e 90 . Foi classificado como pequeno para a idade gestacional aquele cujo peso estivesse abaixo do percentil 10, e grande quando o peso se encontrava acima do percentil 90.

Quando da necessidade de procedimentos de reanimação ao nascer, todas as unidades integrantes da Rede aplicam as normas da Academia Americana de Pediatria adotadas pela Sociedade Brasileira de Pediatria. Dessa forma foi possivel a quantificação do uso de ventilação com balão e máscara, intubação traqueal, massagem cardíaca e/ou medicações utilizadas ${ }^{11}$. O escore de Apgar no $1^{\circ}$ e $5^{\circ}$ minuto de vida foi registrado para todas as crianças $^{12}$.

A análise dos dados foi realizada com os programas Epi-Info 2000 e SPSS 10.0 para Windows. Foram utilizados o teste do $\chi^{2}$ ou exato de Fisher, $t$ de Student, Mann-Whitney, ANOVA com verificação posterior de diferenças de Tukey e regressão logística múltipla.

A análise de regressão logística múltipla foi empregada para determinar as variáveis mais fortemente associadas ao uso antenatal de corticosteóides e às condições de nascimento. No caso da regressão logística múltipla, quanto ao uso de corticosteróides, considerou-se como variável dependente qualquer uso de corticosteróide antenatal (sim ou não). No caso das condições de nascimento, tomou-se o escore de Apgar de $1^{\circ} \mathrm{mi}-$ nuto como variável dependente, classificado como menor que 3 ou maior ou igual a 3. Para todos os testes adotou-se o nível de significância de $5 \%$.

\section{Resultados}

Foram incluidas 463 gestantes e seus 514 recém-nascidos. Dessas, 282 mães (315 neonatos) receberam ao menos uma dose de corticosteróide antenatal e 181 (199 recém-nascidos) não receberam corticosteróide antenatal. Todos os centros utilizaram betametasona, exceto um (unidade 6), que utilizou preferencialmente dexametasona.

A Tabela 1 apresenta a distribuição das gestantes segundo o uso de corticosteróide antenatal e unidade hospitalar onde ocorreu o parto. Podese constatar que houve grande variabilidade de conduta entre as unidades. Computando-se o uso de pelo menos uma dose de corticosteróide antenatal, a terapêutica foi aplicada na proporção de 12,5 a $87,3 \%$ das gestantes. A maneira como a droga foi aplicada às gestantes também foi diferente entre as unidades, ocorrendo grande variabilidade quanto à porcentagem de uso considerado como adequado ${ }^{2}$, variando de 7,8 a $80 \%$ dos tratamentos. Das 123 mães cujo tratamento antenatal com corticosteróide nas diversas unidades foi considerado inadequado, $33(26,9 \%)$ o foram apenas por tempo de tratamento insuficiente (menos de $12 \mathrm{~h}$ antes do nascimento), 28 $(22,7 \%)$ por tempo prolongado (mais de 7 dias antes do nascimento), 30 (24,2\%) por número de ciclos superior ao preconizado e $32(26,0 \%)$ por ambos, muitos ciclos e tempo até o nascimento maior que 7 dias.

Tabela 1 - Distribuição das gestantes segundo o uso de corticosteróide antenatal e a unidade hospitalar onde ocorreu 0 parto.

\begin{tabular}{|c|c|c|c|}
\hline Unidades & $\begin{array}{l}\text { Gestantes } \\
\text { incluídas } \\
\text { no estudo }\end{array}$ & $\begin{array}{c}\text { Gestantes que receberam } \\
\text { corticosteróide* } \\
\text { n ( } \% \text { do total) }\end{array}$ & $\begin{array}{c}\text { Uso adequado } \\
\text { do corticosteróide* } \\
\text { n ( } \% \text { das tratadas) }\end{array}$ \\
\hline 1 & 56 & $7(12,5)$ & $3(42,9)$ \\
\hline 2 & 77 & $55 \quad(71,4)$ & $29 \quad(52,7)$ \\
\hline 3 & 42 & $23(54,7)$ & $15(65,2)$ \\
\hline 4 & 37 & $22(59,5)$ & $16(72,7)$ \\
\hline 5 & 45 & $35(77,8)$ & $28(80,0)$ \\
\hline 6 & 70 & $47 \quad(67,1)$ & $32(68,1)$ \\
\hline 7 & 63 & $55 \quad(87,3)$ & $33(60,0)$ \\
\hline 8 & 73 & $38 \quad(52,0)$ & $3 \quad(7,8)$ \\
\hline Total & 463 & $282(60,1)$ & $159(56,4)$ \\
\hline
\end{tabular}

Entre as gestantes não tratadas previamente com corticosteróide, em $23,7 \%$ das vezes (43/ 181) elas permaneceram internadas por mais de $12 \mathrm{~h}$ previamente ao nascimento da criança. As principais características das gestantes que receberam ou não corticosteróide antenatal estão apresentadas na Tabela 2. Pode-se constatar que foram diferentes algumas das características das gestantes que receberam corticosteróide antenatal de forma adequada ou não, quando comparadas com as que não receberam. O fato de receberem o corticosteróide esteve individualmente associado com maior número de gestações anteriores, de consultas de pré-natal, a maior uso de tocolíticos e com o fato de apresentarem mais freqüentemente hipertensão arterial sistêmica durante a gestação. 
Tabela 2 - Principais características das 463 gestantes incluidas segundo o uso ou não de corticosteróide antenatal.

\begin{tabular}{|c|c|c|c|c|}
\hline & $\begin{array}{l}\text { Uso adequado } \\
\text { corticosteróide }\end{array}$ & $\begin{array}{l}\text { Uso inadequado } \\
\text { de corticosteróide }\end{array}$ & $\begin{array}{c}\text { Sem } \\
\text { corticosteróide }\end{array}$ & $p$ \\
\hline Número & $159(34,3 \%)$ & $123(26,6 \%)$ & $181(39,1 \%)$ & \\
\hline Idade (anos) & $27,3 \pm 7,1$ & $27,4 \pm 7,3$ & $26,1 \pm 6,7$ & $0,17^{*}$ \\
\hline Gestações anteriores & $2,8 \pm 2,5^{\mathrm{a}}$ & $2,4 \pm 1,9$ & $2,21 \pm 2,02^{b}$ & $0,03^{*}$ \\
\hline Filhos vivos & $1,3 \pm 1,8$ & $1,19 \pm 1,31$ & $1,22 \pm 1,62$ & $0,78^{*}$ \\
\hline Consultas de pré-natal & $3,7 \pm 2,6^{b}$ & $4,84 \pm 3,4^{a}$ & $2,41 \pm 2,64^{c}$ & $<0,01^{*}$ \\
\hline Diabetes materno & $6(3,8 \%)$ & $8(6,5 \%)$ & $4(2,2 \%)$ & $0,18^{\#}$ \\
\hline Infecção materna & $41(25,8 \%)$ & $38(30,9 \%)$ & $44(24,3 \%)$ & $0,34^{\#}$ \\
\hline Bolsa rota $>18 \mathrm{~h}$ & $32(20,1 \%)$ & $25(20,3 \%)$ & $35(19,3 \%)$ & $0,99^{\#}$ \\
\hline Hipertensão & $74(46,6 \%)$ & $35(28,4 \%)$ & $37(20,4 \%)$ & $<0,01^{\#}$ \\
\hline Uso de tocolíticos & $40(25,1 \%)$ & $38(30,9 \%)$ & $9(4,9 \%)$ & $<0,01^{\#}$ \\
\hline
\end{tabular}

${ }^{*}$ ANOVA $a>b>c(p<0,05) .{ }^{*} \chi^{2}$. (\%) Relativo ao total no estrato.

Letras-índice não coincidentes indicam diferenças estatisticamente significativas.

Colocando-se as variáveis que apresentaram alguma associação individual com o uso ou não de corticosteróide $(\mathrm{p}<0,2)$ no cálculo da regressão logística múltipla, restaram apenas as seguintes variáveis associadas ao uso antenatal de corticosteróide: número de consultas de pré-natal, hipertensão materna e uso antenatal de tocoliticos. Esses resultados estão apresentados na Tabela 3.

Tabela 3 - Regressão logística múltipla das principais variáveis maternas associadas com o uso antenatal de corticosteróide.

\begin{tabular}{lccc}
\hline & Odds ratio & Intervalo de confiança (95\%) & $\mathbf{p}$ \\
\hline Consultas de pré-natal & 1,23 & $1,13-1,33$ & $<0,01$ \\
Hipertensão & 2,95 & $1,8-4,7$ & $<0,01$ \\
Uso de tocolíticos & 9,29 & $4,3-19,7$ & $<0,01$ \\
\hline
\end{tabular}

As crianças estudadas tiveram, em média, peso de $1.309 \pm 486$ gramas e idade gestacional de $30,3 \pm 2,8$ semanas. Algumas características das crianças cujas mães receberam corticosteróide de forma adequada ou não e das que não receberam o tratamento antenatal estão apresentadas na Tabela 4.

Tabela 4 - Algumas características das crianças cujas mães receberam corticosteróide antenatal de forma adequada ou não e das que não receberam o tratamento.

\begin{tabular}{|c|c|c|c|c|}
\hline & $\begin{array}{l}\text { Uso adequado } \\
\text { de corticosteróide }\end{array}$ & $\begin{array}{l}\text { Uso inadequado } \\
\text { de corticosteróide }\end{array}$ & Sem corticosteróide & $p$ \\
\hline Número de crianças & 157 & 158 & 199 & \\
\hline Peso (g) & $1287 \pm 443^{b}$ & $1417 \pm 434^{a}$ & $1241 \pm 543^{b}$ & $<0,01^{*}$ \\
\hline $\begin{array}{l}\text { Idade gestacional } \\
\text { (semanas) }\end{array}$ & $30,6 \pm 2,2^{\mathrm{a}}$ & $31,1 \pm 2,1^{\mathrm{a}}$ & $29,5 \pm 3,5^{b}$ & $<0,01^{*}$ \\
\hline $\begin{array}{l}\text { Pequenos para idade } \\
\text { gestacional }\end{array}$ & $44(28,0 \%)$ & $42(26,6 \%)$ & $52(26,1 \%)$ & $0,11^{¥}$ \\
\hline Sexo feminino & $77(49,0 \%)$ & $87(55,1 \%)$ & $83(41,7 \%)$ & $0,05^{*}$ \\
\hline Parto cesárea & $106(67,5 \%)$ & $108(68,4 \%)$ & $109(54,8 \%)$ & $0,01^{*}$ \\
\hline
\end{tabular}

$a>b, p<0,05$ para a mesma variável

(\%) Valor porcentual em relação ao total do estrato. $\quad{ }^{{ }^{*}} \chi^{2}$

Letras-índice não coincidentes indicam diferenças estatisticamente significativas. 
Pode-se constatar que as crianças nascidas de mães tratadas ou não com corticosteróide antenatal apresentaram algumas caracteristicas significativamente diferentes. Ao nascer eram crianças pouco mais pesadas (em média cerca de $100 \mathrm{~g})$, com idade gestacional aproximadamente de uma semana maior e mais freqüentemente nascidas por cesareana.
A Tabela 5 apresenta as condições de nascimento e necessidade de cuidados na sala de parto das crianças cujas mães receberam ou não corticosteróide antenatal. As crianças nascidas de mães que receberam corticosteróide antenatal receberam valores mais altos do escore de Apgar no $1^{\circ}$ e $5^{\circ}$ minuto, e menor freqüência de intervenção na sala de parto.

Tabela 5 - Condição ao nascer e necessidade de cuidados na sala de parto das crianças cujas mães receberam ou não corticosteróide antenatal.

\begin{tabular}{|c|c|c|c|c|}
\hline & $\begin{array}{l}\text { Uso inadequado } \\
\text { de corticosteróide }\end{array}$ & $\begin{array}{c}\text { Uso adequado } \\
\text { de corticosteróide }\end{array}$ & $\begin{array}{c}\text { Sem } \\
\text { corticosteróide }\end{array}$ & $p$ \\
\hline Apgar de $1^{\circ}$ minuto & $5,9 \pm 2,5^{a}$ & $6,0 \pm 2,5^{a}$ & $4,5 \pm 2,9^{b}$ & $<0,01^{*}$ \\
\hline Apgar de $5^{\circ}$ minuto & $8,4 \pm 1,8^{a}$ & $8,4 \pm 1,6^{a}$ & $7,1 \pm 2,7^{b}$ & $<0,01^{*}$ \\
\hline \multicolumn{5}{|c|}{ Atendimento na sala de parto } \\
\hline Balão e máscara & $59(37,3 \%)$ & $77(49,0 \%)$ & $91(45,7 \%)$ & $0,1^{¥}$ \\
\hline Intubação & $33(20,9 \%)$ & $38(24,2 \%)$ & $89(44,7 \%)$ & $<0,01^{\ddagger}$ \\
\hline Massagem cardíaca & $4(2,5 \%)$ & $6(3,8 \%)$ & $27(13,6 \%)$ & $<0,01^{¥}$ \\
\hline Drogas & $1(0,6 \%)$ & $4(2,5 \%)$ & $24(12,1 \%)$ & $<0,01 ¥$ \\
\hline
\end{tabular}

$a>b(p<0,05)$ para a mesma variável $\quad{ }^{*}$ ANOVA $\quad{ }^{\sharp} \chi^{2}$

Letras-índice não coincidentes indicam diferenças estatisticamente significativas.

Com a finalidade de se verificar, por meio de regressão logística, quais variáveis estariam associadas à condição de nascimento, tomou-se o escore de Apgar de $1^{\circ}$ minuto como variável dependente, classificado como menor que 3 ou maior ou igual a 3. Avaliou-se quais variáveis estariam associadas individualmente ao escore de Apgar de $1^{\circ}$ minuto. As variáveis número de consultas de pré-natal, hipertensão materna, uso de corticosteróide (agrupados adequado e inadequado), uso de tocolíticos, tipo de parto, idade gestacional, peso de nascimento e gênero entraram no modelo de regressão logística e dessas permaneceram como estatisticamente significantes as apresentadas na Tabela 6.

Tabela 6 - Regressão logística múltipla das variáveis associadas com os valores de Apgar de $1^{\circ}$ minuto (Apgar $<3=0 ;$ Apgar $\geq 3=1$ ).

\begin{tabular}{lccc}
\hline Variável & $\begin{array}{c}\text { Odds } \\
\text { ratio }\end{array}$ & $\begin{array}{c}\text { Intervalo de } \\
\text { confiança (95\%) }\end{array}$ & $\mathbf{p}$ \\
\hline $\begin{array}{l}\text { Uso antenatal } \\
\text { de corticosteróide* }\end{array}$ & 2,47 & $1,43-4,30$ & $<0,01$ \\
$\begin{array}{l}\text { Idade gestacional } \\
\begin{array}{l}\text { Pequeno para } \\
\text { idade gestacional }\end{array}\end{array}$ & 1,38 & $1,19-1,60$ & $<0,01$ \\
\hline
\end{tabular}

${ }^{*}$ Considerou-se uso adequado e inadequado da droga.
Pode-se constatar que de todas as variáveis individualmente associadas, apenas permaneceram o uso antenatal de corticosteróide, a idade gestacional e o fato de ser pequeno para a idade gestacional. As três variáveis apresentaram efeito protetor, ou seja, aumentaram a probabilidade de o escore de Apgar ser maior que três no primeiro minuto.

\section{Discussão}

A despeito das inúmeras evidências de seus benefícios ${ }^{4}$, o uso antenatal de corticosteróides nas gestações em risco de término prematuro ainda não é universalmente difundido entre as instituições universitárias participantes do estudo.

Em princípio todas as gestações em risco de término prematuro deveriam receber o corticosteróide antenatal. No entanto, nossas unidades encontram-se muito longe desse ideal. Somente em três das oito unidades avaliadas o corticosteróide foi aplicado em mais de $70 \%$ das gestantes e surpreendentemente, em um dos hospitais, a porcentagem de uso foi de apenas $12,5 \%$ das mães. A ampla variação de freqüência de uso entre os hospitais, semelhante à encontrada no presente estudo, também foi verificada entre serviços internacionais ${ }^{13,14}$. Deve-se pon- 
derar que, além da baixa freqüência do uso do corticosteróide antenatal, em quase metade das vezes o uso da terapêutica foi diferente do esquema recomendado ${ }^{2}$.

A inadequação terapêutica mais freqüentemente detectada foi o intervalo de tempo entre a aplicação da última dose e o nascimento da criança. Evidentemente essa variável em parte está fora do controle dos obstetras, o que é reforçado pelo fato de ter ocorrido de forma indistinta entre as oito unidades. No entanto, o mesmo não pode ser dito quanto à administração de mais ciclos da droga que o preconizado. Esse mau uso do corticosteróide ocorreu em cerca de um quarto das vezes em que foi utilizado, chegando a $78 \%$ das gestantes tratadas no Hospital ${ }^{8}$. Hoje a literatura já apresenta diversas evidências de que ciclos múltiplos de corticosteróide antenatal não apresentam resultados superiores ao ciclo único e podem vir a ser deletérios para o recém-nascido $^{15,16}$. Sem dúvida, é muito importante que as rotinas dos serviços avaliados, especialmente por se tratarem de instituições formadoras de opinião no cenário brasileiro, sejam constantemente revistas à luz dos novos conhecimentos disponiveis.

Avaliou-se o tempo decorrido entre a internação da gestante e o nascimento da criança. O objetivo dessa análise foi verificar se alguma paciente poderia, mesmo após a internação, ter recebido o corticosteróide em tempo de promover alguma proteção a seu concepto. Em $23,7 \%$ das vezes $(43 / 181)$ as mães permaneceram internadas por mais de 12 horas previamente ao nascimento da criança, chegando em alguns casos a dias de internação. Nessas situações, ao menos teoricamente, teria havido tempo hábil para o estabelecimento da terapia com corticosteróide, podendo representar mais uma oportunidade perdida de auxílio ao futuro recém-nascido.

A pergunta que se segue é porque serviços médicos de primeira linha no cenário nacional não estão utilizando amplamente o corticosteróide antenatal, já que essa é uma prática confirmada como eficaz ${ }^{4}$. Uma justificativa que informalmente se costuma utilizar é que a população atendida pelos hospitais universitários é de baixo nível social e, dessa forma, tem dificuldade de acesso, ou mesmo, espontaneamente, não participa do seguimento ambulatorial pré-natal. Em outras palavras, essas pacientes não oferecem a oportunidade de receber o tratamento. Pelo menos em parte esse fato deve ter sua parcela de responsabilidade nos resultados encontrados. No entanto, se fosse totalmente verdadeiro, a porcentagem de tratamentos seria uniforme entre as unidades, o que não foi.
Por outro lado, as caracteristicas socioeconômicas maternas não justificam as diferenças de uso de corticosteróide antenatal detectado entre as oito unidades. Apesar de não dispormos de dados a respeito do nivel sociocultural da população estudada, não vemos razão que justifique haver diferenças a esse respeito entre as pacientes atendidas pelas oito instituições estudadas, já que são todos hospitais universitários prestadores de serviço ao Sistema Único de Saúde. Acreditamos que a grande variabilidade encontrada entre as instituições estaria realmente relacionada às rotinas médicas empregadas nas Unidades. Se esse é o caso, é maior a possibilidade de que venha a ser facilmente sanado, estando o emprego mais amplo da terapêutica na dependência da simples mudança das rotinas locais.

As caracteristicas das gestantes que receberam corticosteróide não são semelhantes às das que não receberam, indicando haver fator materno envolvido. A regressão logística das variáveis maternas associadas ao uso de corticosteróide antenatal indicou que o número de consultas no pré-natal, a presença de hipertensão arterial materna e o uso antenatal de tocolíticos apresentaram relação direta com o uso do corticosteróide. Pode-se depreender que as mães que receberam corticosteróide antenatal foram mais bem assistidas que as que não o receberam, sendo possivelmente o uso antenatal de corticosteróide parte dessa melhor assistência oferecida a essas mulheres.

Também deve ser ressaltado que o uso de corticosteróide antenatal não foi influenciado por situações clínicas maternas em que o seu uso, ainda hoje, gera polêmicas, como no caso do diabete materno ou ruptura prematura das membranas ${ }^{17}$. A hipertensão materna, que já foi considerada contra-indicação ao uso de corticosteróide ${ }^{1,17,18}$, foi associada, mesmo após a regressão logística, ao maior uso da droga. Uma justificativa seria que essas mães, possivelmente sendo acompanhadas mais de perto durante o pré-natal, tiveram maior oportunidade de receber o corticosteróide.

Somente uma das unidades avaliadas fez uso preferencial da dexametasona. Betametasona e dexametasona são os corticosteróides que têm sido utilizados preferencialmente no tratamento antenatal visando a aceleração do amadurecimento fetal. Ambos atravessam a barreira placentária e possuem ação biológica praticamente idêntica ${ }^{19}$. Meta-análise dos estudos randomizados demonstrou que ambas as drogas diminuem a freqüência da síndrome da dificuldade respiratória, porém somente a betametasona diminuiu a mortalidade 
fetal $^{20}$. Existe a sugestão proveniente de estudo retrospectivo e não controlado de que a betametasona pode reduzir aproximadamente pela metade a incidência de leucomalácia periventricular em relação às gestantes não tratadas ou tratadas com dexametasona ${ }^{21}$. Os filhotes de ratas tratadas com betametasona tiveram desenvolvimento neurológico melhor que as tratadas com dexametasona ${ }^{22}$. No entanto, o consenso de 2000 do National Institute of Child Health and Human Development ${ }^{3}$ revisou esse tópico e não considerou que as evidências científicas eram fortes o bastante para recomendar exclusivamente o uso de betametasona. O Comitê do Colégio Americano de Ginecologia e Obstetrícia, em 2002, também não apontou nenhuma preferência entre os dois corticosteróides ${ }^{2}$.

As crianças cujas mães receberam corticosteróide antenatal apresentaram melhores condições ao nascimento. Isso pôde ser constatado pelos valores do escore de Apgar no $1^{\circ}$ e $5^{\circ} \mathrm{mi}-$ nuto. $\mathrm{O}$ efeito protetor do corticosteróide, assim como da idade gestacional e do retardo de crescimento intra-uterino, permaneceram após regressão logística, como já fora demonstrado anteriormente por outros autores ${ }^{23}$.

Interessante notar que alguns autores não consideram o escore de Apgar adequado para avaliar a condição de nascimento em pré-termos. Isso se deve ao fato de que o escore inclui entre seus cinco componentes três que são dependentes de maturidade (esforço respiratório, irritabilidade reflexa e tônus muscular) ${ }^{24,25}$. No entanto, quando se avaliou a necessidade de intervenção na sala de parto, ficou claro que de fato as crianças cujas mães receberam corticosteróide nasceram em melhores condições. Elas necessitaram de menos condutas agressivas na sala de parto como intubação traqueal, massagem cardiaca ou uso de adrenalina. $O$ fato de favorecer melhores condições de nascimento é importante benefício da terapia antenatal com corticosteróide.

Concluindo, pode-se dizer que o uso de corticosteróide antenatal nas instituições estudadas ocorreu na maioria dos centros em freqüência abaixo da desejada e em metade dos casos foi feito de forma inadequada. O tratamento foi positivamente associado ao número de consultas de pré-natal, uso antenatal de tocolíticos e presença de hipertensão materna, sugerindo serem mães mais bem acompanhadas durante o pré-natal. O uso de corticosteróide antenatal (adequado ou não) associou-se a melhores condições de nascimento, o que representa importante vantagem inicial para o prognóstico desses pequenos prematuros. Os resultados indicam a necessidade de se refor- çar a importância e criar mecanismos para estimular o uso antenatal de corticosteróide nas gestações de risco de parto prematuro.

\section{ABSTRACT}

Purpose: to assess the use of antenatal corticosteroid (AC) by mothers and its repercussion on the birth conditions of preterm babies at the eight university neonatal units belonging to the Brazilian Network of Neonatal Research. Methods: an observational prospective cohort study. All 463 pregnant women with a gestational age (GA) of 23 to 34 weeks and their 514 newborn babies were evaluated during the period from August 1 to December 31, 2001. The data were obtained by maternal interview, by the analysis of the medical records and by the follow-up of the newborn infants, and analyzed statistically using $\chi^{2}$, Mann-Whitney and ANOVA tests and multiple logistic regression, with the level of significance set at 0.05 .

Results: 60.1\% (282/463) of the pregnant women (a variation from 12.5 to $87.3 \%$ among units) received at least one $A C$ dose. The AC use was directly associated with the number of prenatal visits, with maternal hypertension and with the antenatal use of tocolytic agents. Babies from treated pregnant women presented higher birth weight $(1,379 \pm 421$ vs $1,244 \pm 543 \mathrm{~g})$, longer gestational age $(30.9 \pm 2.0 \mathrm{vs}$ $29.5 \pm 3.5$ weeks), better Apgar scores at the 1st and 5th minute, and a reduced need for intervention in the delivery room. The use of $A C$, the GA and a baby small for $G A$ independently improved the birth conditions.

Conclusions: at most centers, AC was administered at frequencies below the desired ones, and in 50\% of cases in an inadequate manner. Treatment was applied more to mothers who received appropriate prenatal care and was associated with better birth conditions.

KEYWORDS: Antenatal glucocorticoids. Prematurity. Low birth weight.

\section{Referências}

1. Liggins GC, Howie RN. A controlled trial of antepartum glucocorticoid treatment for prevention of the respiratory distress syndrome in premature infants. Pediatrics 1972; 50:515-25.

2. Committee on Obstetric Practice. ACOG committee opinion: antenatal corticosteroid therapy for fetal maturation. Obstet Gynecol 2002; 99: 871-3.

3. Antenatal corticosteroids revised: repeat courses. NIH Consens Statement 2000; 17:1-18.

4. Crowley P. Prophylactic corticosteroids for preterm birth. Cochrane Database Syst Rev 2000; (2):CD000065. 
5. Crowley P, Chalmers I, Keirse MJ. The effects of corticosteroid administration before preterm delivery: an overview of the evidence from controlled trials. Br J Obstet Gynaecol 1990; 97:11-25.

6. Albuquerque ICC, Amorim MMR, Meneses J, Katz L, Santos LC. Avaliação do impacto da corticoterapia antenatal para a aceleração da maturidade pulmonar fetal nos recém-nascidos em maternidade-escola brasileira. Rev Bras Ginecol Obstet 2002; 24:655-61.

7. Silva LK, Costa TP, Reis AF, Iamada NO, Azevedo $\mathrm{AP}$, Albuquerque CP. Avaliação da qualidade da assistência hospitalar obstétrica: uso de corticóides no trabalho de parto prematuro. Cad Saúde Pública 1999; 15:817-29.

8. Chung CS, Myrianthopoulos NC. Congenital anomalies: mortality and morbidity, burden and classification. Am J Med Genet 1987; 27:505-23.

9. Ballard JL, Khoury JC, Wedig K, Wang L, EilersWalsman BL, Lipp R. New Ballard Score, expanded to include extremely premature infants. J Pediatr 1991; 119:417-23.

10.Alexander GR, Himes JH, Kaufman RB, Mor J, Kogan M. A United States national reference for fetal growth. Obstet Gynecol 1996; 87:163-8.

11.International Guidelines for Neonatal Resuscitation: An excerpt from the Guidelines 2000 for Cardiopulmonary Resuscitation and Emergency Cardiovascular Care: International Consensus on Science. Contributors and Reviewers for the Neonatal Resuscitation Guidelines. Pediatrics 2000; 106:E29.

12.Apgar V. A proposal for a new method of evaluation of the newborn infant. Curr Res Anesth Analg 1953; 32:260-7.

13. Ryan CA, Finer NN. Antenatal corticosteroid therapy to prevent respiratory distress syndrome. J Pediatr 1995; 126:317-9.

14.Chien LY, Ohlsson A, Seshia MM, et al. Variations in antenatal corticosteroid therapy: a persistent problem despite 30 years of evidence. Obstet Gynecol 2002; 99:401-8.

15.Meneguel JF, Guinsburg R, Miyoshi MH, Peres CA, Kopelman BI, Camano L. Corticosteróide antenatal: ciclo único versus múltiplo - comparação de resultados. Rev Bras Ginecol Obstet 2002; 24:505-10.

O trabalho foi desenvolvido nas seguintes instituições: Berçário Anexo à Maternidade; Universidade de São Paulo (USP/RP) / Centro de Atenção Integral à Saúde da Mulher (CAISM); Universidade Federal do Rio Grande do Sul (UFRGS) / Escola Paulista de Medicina (EPM)/ Faculdade de Medicina de Botucatu; Universidade Estadual de Campinas (UNICAMP) / Fundação Oswaldo Cruz (FIOCRUZ)/ Hospital das Clínicas de Ribeirão Preto / Hospital das Clínicas de São Paulo / Hospital de Clínicas de Porto Alegre; Universidade Federal de São Paulo (UNIFESP) / Hospital São Lucas; Universidade Estadual Paulista (UNESP) / Hospital São Paulo; Universidade de São Paulo (USP/SP) / Instituto Fernandes Figueira; Pontificia Universidade Católica do Rio Grande do Sul (PUCRS)
16.Banks BA, Macones G, Cnaan A, et al. Multiple courses of antenatal corticosteroids are associated with early severe lung disease in preterm neonates. J Perinatol 2002; 22:101-7.

17.Bloom SL, Leveno KJ. Corticosteroid use in special circumstances: preterm ruptured membranes, hypertension, fetal growth restriction, multiple fetuses. Clin Obstet Gynecol 2003; 46:150-60.

18. Sass N, Cançado RR, Oliveira ML, Torloni MR. Corticoterapia pré-natal nas síndromes hipertensivas da gestação e seus efeitos na pressão arterial materna. Rev Assoc Med Bras 2001; 47:255-8.

19.Fanaroff AA, Hack M. Periventricular leukomalaciaprospects for prevention. N Engl J Med 1999; 341:1229-31.

20.Ballard PL, Ballard RA. Scientific basis and therapeutic regimens for use of antenatal glucocorticoids. Am J Obstet Gynecol 1995; 173:25462.

21.Baud O, Foix-L'Helias L, Kaminski M, et al. Antenatal glucocorticoid treatment and cystic periventricular leukomalacia in very premature infants. N Engl J Med 1999; 341:1190-6.

22.Rayburn WF, Christensen HD, Gonzalez CL. A placebocontrolled comparison between betamethasone and dexamethasone for fetal maturation: differences in neurobehavioral development of mice offspring. Am J Obstet Gynecol 1997; 176:842-50.

23.Gardner MO, Goldenberg RL, Gaudier FL, Dubard MB, Nelson KG, Hauth JC. Predicting low Apgar scores of infants weighing less than 1000 grams: the effect of corticosteroids. Obstet Gynecol 1995; 85:170-4.

24.Catlin EA, Carpenter MW, Brann BS 4th, et al. The Apgar score revised: influence of gestational age. J Pediatr 1986; 109:865-8.

25.Stark CF, Gibbs RS, Freedman WL. Comparison of umbilical artery $\mathrm{pH}$ and 5-minute Apgar score in the low-birth-weight and very low-birth-weight infant. Am J Obstet Gynecol 1990; 163:818-23.

Auxílio financeiro: Apoio do Ministério da Saúde. 